\title{
Does the solar granulation change with the activity cycle?
}

\author{
R. Muller ${ }^{1}$, A. Hanslmeier ${ }^{2}$, D. Utz ${ }^{2}$, and K. Ichimoto ${ }^{3}$ \\ 1 Institut de Recherche en Astrophysique et Planétologie de l'Observatoire Midi-Pyrénées, Université de Toulouse, CNRS, \\ section Observatoire du Pic du Midi, 57 Avenue d'Azereix, 65008 Tarbes, France \\ e-mail: muller@ast.obs-mip.eu \\ 2 Institut für Physik, Karl-Franzen Universität Graz, Universitätsplatz 5, 8010 Graz, Austria \\ e-mail: arnold.hanslmeier@uni-graz.at \\ 3 Kwasan and Hida Observatories, Kyoto University, Gifu, Japan
}

Received 11 October 2017 / Accepted 5 April 2018

\begin{abstract}
Context. Knowledge of the variation of the solar granulation properties (contrast and scale) with the 11-yr activity cycle is useful for a better understanding of the interaction between magnetic field and convection at global or local scales. A varying granulation may also contribute to irradiance variations and affect the p-mode damping rates and lifetimes.

Aims. HINODE/SOT blue continuum images taken in the frame of the synoptic program at the disk center on a daily basis between November 2006 and February 2016 are used. This period covers the minimum of activity between cycles 23 and 24 and the maximum of cycle 24 .

Methods. The sharpness of a significant number of images was reduced because of instrumental aberrations or inaccurate focusing Only the sharpest images were selected for this investigation.

Results. To be detectable with HINODE/SOT images, the variation of the granulation contrast and of the granulation scale at the disk center should have been larger than 3\%. As it is not the case, it is concluded that they varied by less than $3 \%$ through the weak cycle 24 .
\end{abstract}

Key words. Sun: granulation - Sun: activity - dynamo

\section{Introduction}

Some models of the structure and evolution of the Sun have been constructed which include variable magnetic fields and turbulence, in order to explain the variation of some global parameters through the solar cycle (Li et al. 2003, 2009; Mullan et al. 2007). Indeed, observations have revealed that the total solar irradiance (e.g., Wilson \& Hudson 1991; Fröhlich 2000; Solanki et al. 2013), the solar effective temperature (Kuhn et al. 1988; Kuhn \& Schüssler 2000) and the solar diameter (the optical diameter derived from limb profiles, Emilio et al. 2007) as well as the seismic diameter derived from observed shifts in f-modes and which measures a diameter several thousands of kilometers below the surface (Li et al. 2003, 2009; Lefebvre \& Kosovichev 2005; Emilio et al. 2007) vary over the solar cycle. Variations in turbulence and magnetic field are often invoked to explain observed p-mode frequency shifts during the solar cycle (Basu \& Antia 2000; Kuhn \& Schüssler 2000; Dziembowski \& Goode 2005). Another consequence of a varying turbulence could be the change of the p-mode damping rates (Houdek et al. 2001) and lifetimes (Burtseva et al. 2009) due to the change of the length scale of convective eddies. Magnetic field manifests itself at the surface of the Sun as sunspots, as tiny bright magnetic elements which form the photospheric network at the boundaries of supergranules and as small bi-polar loops at the granular scale inside the network. The 11-yr activity cycle is mainly due to the emergence of sunspots through the photosphere, produced by the solar dynamo with a periodic rate, while the network and intra-network magnetic fluxes are relatively stable over the cycle, at least at the disk center (Buehler et al. 2013; Utz et al. 2016). Magnetic field and turbulence can interact either at a global scale (Li et al. 2003, 2009) or at a local scale (Cattaneo et al. 2003; Vögler et al. 2005).

The presence of time-varying magnetic fields in the solar interior causes changes in the structure of the Sun, which manifest themselves in the variations of global and helioseismic parameters (Libbrecht \& Woodard 1990; Parker 1995; Li et al. 2003; Lefebvre \& Kosovichev 2005; Mullan et al. 2007). Therefore one may also expect to see changes in the properties of the convective cells visible at the surface. Because of its large contrast, the solar granulation is the most easily visible signature of convection. Although observations (Hathaway et al. 2000; Rieutord et al. 2008) as well as numerical simulations (De Rosa et al. 2002; Norlund et al. 2009) indicate that there is a continuous spectrum of motions of all scales, from global to subgranular, two more convective patterns are observed at the surface of the Sun, the mesogranulation (November \& Simon 1988 ) in the range of sizes on the order $3-10 \mathrm{Mm}$ and the supergranulation (Leighton et al. 1962) in the range on the order $10-50 \mathrm{Mm}$. Their contrast is, however, very weak, making these convective patterns detectable only indirectly, thanks to their associated horizontal and vertical velocity fields and to concentrations of magnetic elements at their boundaries. However, the power spectra of kinetic energy show distinct peaks only at the scale of granulation and supergranulation, but not at the scale of mesogranulation. In numerical simulations the spectrum of motions is continuous in the range covering granular and mesogranular scales (Norlund et al. 2009, and references therein). The solar convection thus appears to be multiscale in nature, ranging from subgranular to supergranular cell sizes, and beyond to giant cells (Norlund et al. 2009). Among the observable convective patterns, granulation is the best suited to investigate solar cycle 


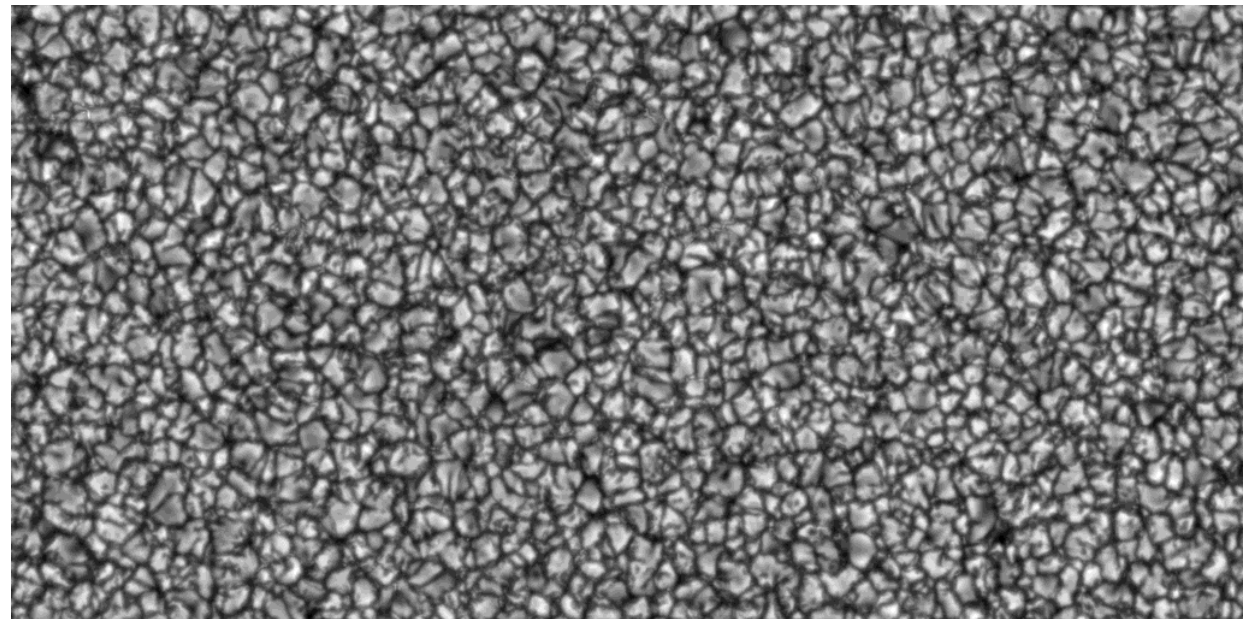

Fig. 1. A $1024 \times 512\left(114^{\prime \prime} \times 57^{\prime \prime}\right)$ subfield of a $2048 \times 1024\left(228^{\prime \prime} \times 114^{\prime \prime}\right)$ blue continuum HINODE/SOT image from the synoptic program. related variations of turbulent convection, thanks to its large contrast and because its observation is now relatively easy from the space, and from the ground as well thanks to adaptive optics and efficient image restoration techniques. Although the granulation size distribution, which decreases continuously from the smallest currently observable granules (on the order of $100 \mathrm{~km}$ ) to the largest ones (on the order of $3000 \mathrm{~km}$, Roudier \& Muller 1986), one can derive a median scale from the power spectrum of the intensity fluctuations. This has been used to investigate the variation of the granulation scale along the solar cycle by Muller et al. (2007, 2011) and it is also used in this paper. The granulation contrast is derived from the power spectrum of the intensity fluctuations too. The result of such an investigation should help us to understand better the interaction between turbulence and magnetic field, and should provide an additional constraint for modeling the solar activity cycle.

The variation of the solar granulation over the solar cycle has been little investigated because of the lack of sets of images of homogeneous spatial resolution. Most published results are derived from images taken at the Pic du Midi Observatory. The first one was published by Macris \& Rösch (1983) based on images taken at the Pic du Midi Observatory. They found that the mean distance between granules decreases with increasing activity. But the set of images they used was not homogeneous, because they were taken with two different refractors, of 38 and $50 \mathrm{~cm}$ of diameter, at different wavelengths, and under slightly different seeing conditions. Later, Macris et al. (1984), Roudier (1986) and Muller (1988), using more homogeneous sets of high resolution images, all taken with the $50 \mathrm{~cm}$ refractor at the Pic du Midi Observatory and at the same wavelength, counted the number of granules per surface unit, and found that it increases with increasing magnetic activity. All these results, obtained with granules identified visually, showed that the granulation scale varies in antiphase with the number of sunspots. New results have been published by Muller et al. (2007), based on the sets of images from the Pic du Midi Observatory used in the previous investigations, which started in 1978, and which was then extended to 1993. The scale and the contrast were both derived from power spectra computed on the digitized images. The variation of the granulation scale related to the solar cycle reported in the previous papers was not confirmed; but it was found that the granulation contrast varies nearly in phase with the solar cycle, being lower at maxima of activity. It thus appears that the variation of the granulation over the 11-yr solar cycle is still an open issue which deserves to be investigated further with very homogeneous sets of images.
High resolution images of the solar granulation free of atmospheric perturbations, regularly produced by the $50 \mathrm{~cm}$ Solar Optical Telescope (SOT) on-board the satellite HINODE between November 2006 and February 2016, are now available. These images have been used in this paper to investigate the variation of the solar granulation over the activity cycle with more accuracy than before.

After a presentation of the HINODE/SOT synoptic program in Sect. 2, the method of analysis of the blue continuum images is described in Sect. 3. The results are presented in Sect. 4 and discussed in Sect. 5.

\section{Observations: HINODE/SOT synoptic program}

The HINODE synoptic program provided broadband images obtained at the disk center with the Optical Solar Telescope (e.g., Tsuneta et al. 2009). They were taken a few seconds apart in blue, green and red continuum windows at $450.45,555.05,668.40 \mathrm{~nm}$ respectively, in the $G$-band lines of the $\mathrm{CH}$ molecule at $430.50 \mathrm{~nm}$, in the $\mathrm{CN}$ band head at $388.35 \mathrm{~nm}$ and in the $\mathrm{Ca}$ II $\mathrm{H}$ line at $396.85 \mathrm{~nm}$, in general once or twice a day. In this work we have only used the blue continuum images. During the observations, the focus position was controlled in the $G$-band; when optimized in this wavelength, it is also optimized in the nearby blue continuum $0.4 \mathrm{~nm}$ window. But unfortunately, this set of images is not as homogeneous as expected, because a significant number of them appear to be blurred to a varying degree; the cause of blurring may be inaccurate focusing or/and optical aberrations as one will see in Sect. 4. Consequently, great care is taken in this investigation to only select the sharpest images, in order to get the most homogeneous set of high resolution granulation images currently available, spanning nearly one solar cycle (although the minimum of activity between cycle 23 and cycle 24 was longer than usual). Before February 2008, when a serious telemetric problem occured, between two and four images were taken every day in each wavelength; after that month and until September 2009 the cadence was reduced to about one image taken every two days, but then on increased again to a cadence of about one observation per day. Two adjacent cameras with $2048 \times 2048$ square pixels of nominal scale $0 .{ }^{\prime \prime} 055$, took rectangular images of nominal size 228 $" \times 114 "$. We have taken into account the annual variation of the Earth-Sun distance by changing the pixel scale accordingly. After the telemetric problems which occurred in January 2008 , the images of the synoptic program were binned by a factor of two. Fortunately, this is not a problem in this work, since the images taken before this date were binned by a factor of two for 
R. Muller et al.: Variation of the solar granulation

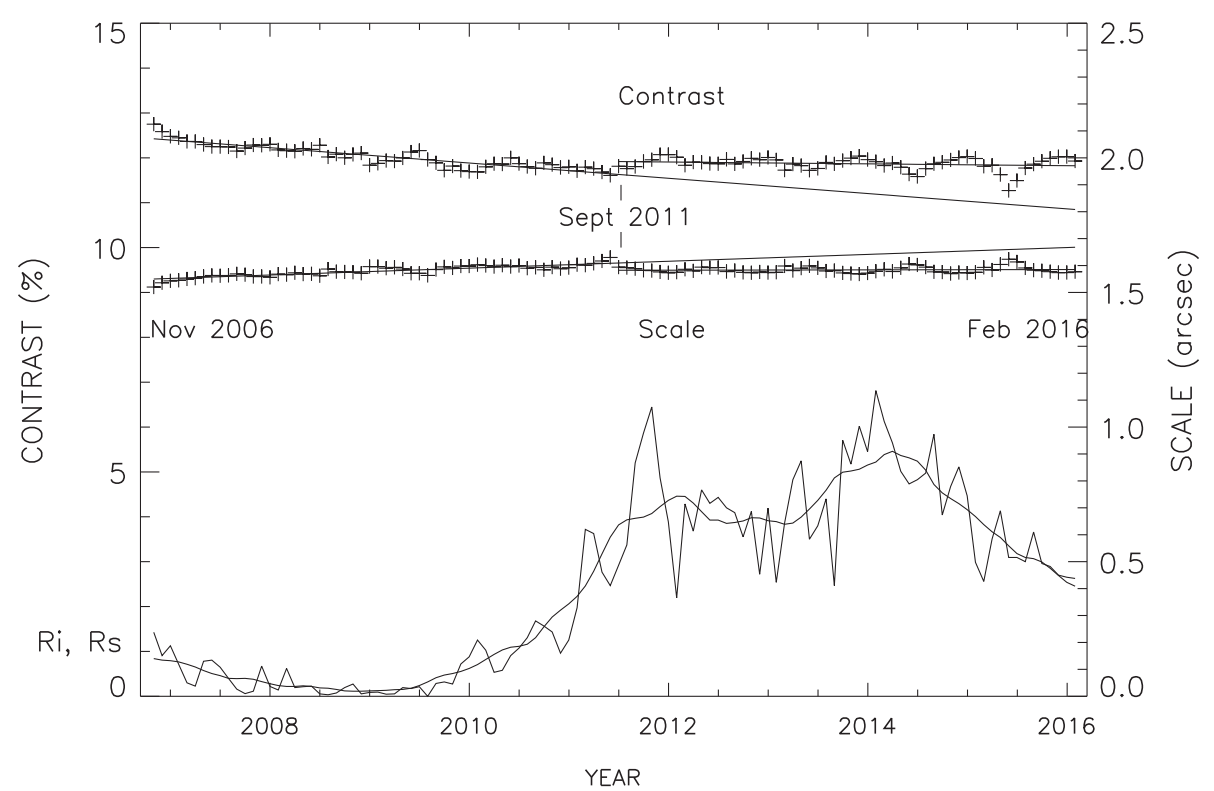

Fig. 2. Variation of the granulation contrast $(+)$ and scale $(+)$ at the disk center, corrected from the annual variation of the distance Earth-Sun, between November 2006 and February 2016; each cross represents a monthly average. Linear trends before and after September 2011 are overplotted. Ri and Rs are the Monthly Sunspot Index and the Smoothed Sunspot Index respectively, published by the SIDC (of the Royal Observatory of Belgium), divided by 20 in order to enter into the Contrast $y$-axis of the figure. analysis. So the size of all the analyzed images is $2048 \times 1024$ pixels. The program ended in February 2016, when both cameras broke down. All images were calibrated with the standard solar reduction software distributed under SSWIDL, which corrects for bad pixels, readout errors, dark current and flat field. A $1024 \times 512$ pixel subfield of a calibrated image is shown in Fig. 1. In each image, the left and the right square subfields are analyzed separately; this helps us, by comparing the results in each of them, to disentangle solar variations from instrumental effects, which is crucial for our investigation (see the next section and Muller et al. 2011).

\section{Granulation images analysis}

As mentioned in Sect.2, a significant number of images from the synoptic program appear to be blurred to a varying degree. In order to get a reliable variation, the sharpest images must be selected, and only these ones used for further analysis. About $25 \%$ of the images have been actually removed because of blurring. The method of selection is described in detail in Muller et al. (2011) and only a summary is presented here. In a first step, the images containing dark columns, sunspots pores, abnormal granulation ${ }^{1}$ were removed visually; the images which clearly appear out of focus were also removed visually.

In the second step the granulation contrast and scale of the selected images were derived from the power spectrum (Muller et al. 2007, 2011), separately in the left and right half fields of view of size $114^{\prime \prime} \times 114^{\prime \prime}$. The root mean square of the intensity fluctuations, Irms, which, for simplicity, we refer to as contrast, is the square root of the integral, above the noise level, of the power spectrum in the granulation range, between $0 .{ }^{\prime \prime} 3$ (in order to remove the high frequency noise) and 4 " (in order to remove non-granular larger scale fluctuations, like the 5-min oscillations and the mesogranulation). The scale is defined as the inverse of the mean wavenumber of the power spectrum in the granulation range, converted in arcseconds. The contrast and the scale derived in this way are about $12 \%$ and about $1 .^{\prime \prime} 6(1160 \mathrm{~km})$ respectively.

When the contrast of the left field-of-view (FOV) of the selected images is plotted versus the contrast of the right FOV, they

\footnotetext{
1 Abnormal granulation is a granulation area which appears smeared and where the contrast is reduced by the presence of many magnetic bright points in the intergranular lanes; in the quiet sun they belong to the remnants of an active region.
}

appear correlated: when the contrast is large (small) in one FOV it is also large (small) in the other FOV (see Fig. 5 in Muller et al. 2011, which shows the correlation for the images selected in January 2007). But as the granulation evolves independently over time in each FOV, they should not be correlated. The correlation is certainly due to an instrumental blurring which is not stable over time and which thus may affect each image to a different degree. A similar correlation is found for the scale. Figure 6 in Muller et al. (2011) also shows that the variation of the contrast is anti-correlated with the variation of the scale. This is also expected when the images are blurred with a varying point spread function. The final image selection procedure is described in detail in Muller et al. (2011). It is based on the difference between the granulation contrast and the granulation scale of the analyzed images, which increases with increasing blurring. In Fig. 7 of Muller et al. (2011), the contrast and the scale of the selected images of January 2007 are both normalized to 1 (i.e., their mean monthly contrast and their mean monthly scale are both set to one). The amplitude of the difference between the normalized scale and the normalized contrast of the images is a function of their sharpness. A threshold of 0.035 for this difference is used to select the sharpest images.

\section{Results}

The monthly averages of the granulation contrast and scale, computed with the sharpest selected images, are plotted in Fig. 2, together with the Monthly Sunspot Index and the Smoothed Sunspot Index, published by the SIDC (Solar Influence Data Analysis Center, Royal Observatory of Belgium, Brussels), from November 2006 to February 2016. This period covers the end of the declining phase of cycle 23 , the extended minimum between cycles 23 and 24 (2008-2010), the rising phase of cycle 24 , its maximum and the beginning of its declining phase. It is noticeable that during the period covered by the observations the behavior of the solar cycle was unusual, with an extended minimum and a low maximum of activity.

The granulation contrast is found to decrease and the scale to increase steadily from November 2006 to September 2011, by $11 \%$ and $6 \%$ respectively (Fig. 2). It jumps a little bit between September 2011 and January 2012 before appearing stabilized to a nearly constant value after January 2012. 

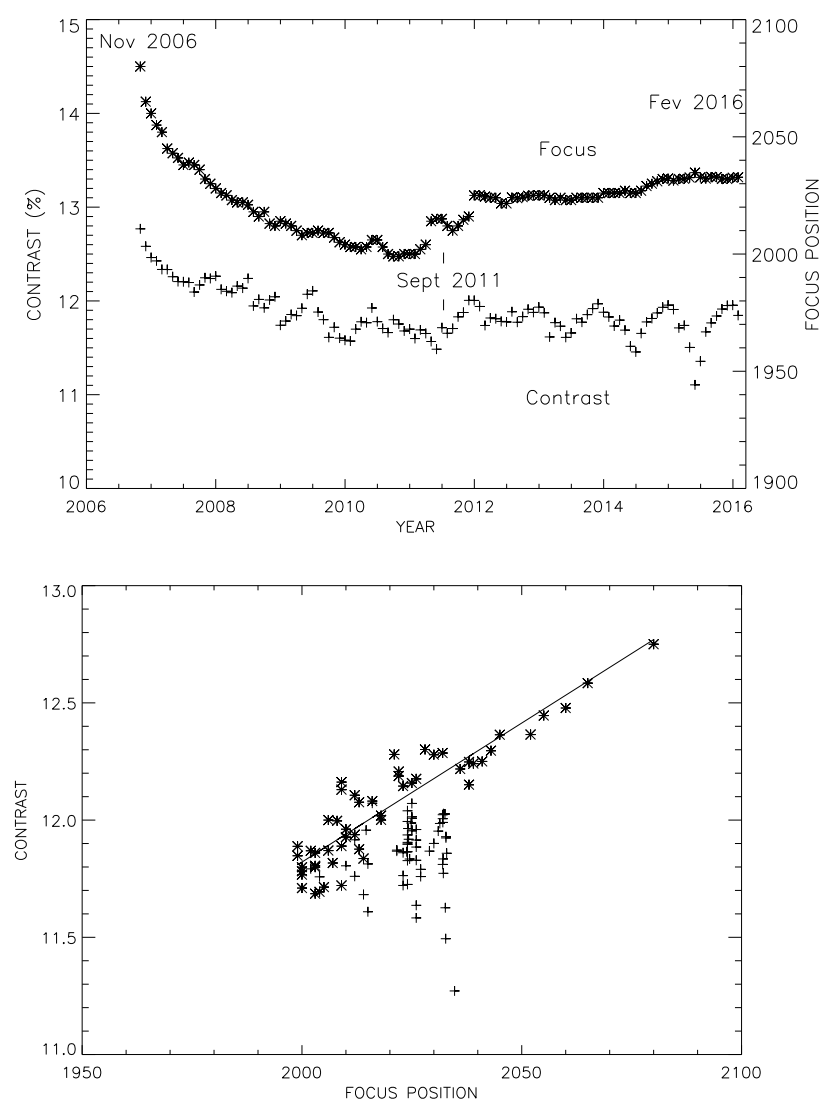

Fig. 3. Top: variation of the granulation contrast $(+)$ and of the focus position (*) of the selected images, during the period of observation November 2006-February 2016; each cross and each star represents a monthly average. Bottom: correlation between contrast and focus position. (*): for the period November 2006-December 2010; (+): for the period January 2011-February 2016.

This long-term trend is related to temperature variations of both the main mirror and of the heat dump mirror support which both increased from the beginning of the HINODE operation to September 2011 and have then been more or less stabilized; the reduction of micro-vibrations of mirrors in September 2011 has slightly improved the sharpness of the images (Ichimoto 2016, priv. comm.). The one-year period modulation of the contrast variation, still clearly visible in Fig. 2 although it has been corrected from the annual variation of the distance Earth-Sun, appears to be in phase with the annual temperature variation of the main mirror (Hinode technical report, 2016). Thus the mirror temperature and the granulation contrast are both minimum during the eclipse season around July. Moreover, during the first days of July, Earth is at its largest distance from the Sun. The eclipse season and the reduced insolation combine to reduce the satellite mirror temperature, thus increasing in width the PSF (and reducing the spatial resolution accordingly). The larger distance also reduces the spatial resolution. All these effects contribute to reduce the contrast of the solar granulation in July. But the distance change is the same every year and alone, it cannot explain the strong contrast variations during the last three years of observations, which remain not well understood. The variation of the granulation scale is in antiphase to that of the contrast, as expected for variations of instrumental origin (namely, variations of the mirror temperature which induce optical aberrations and focal length variations).

The focus position of each image can be found in the associated header. During the nearly ten years of observations the focus

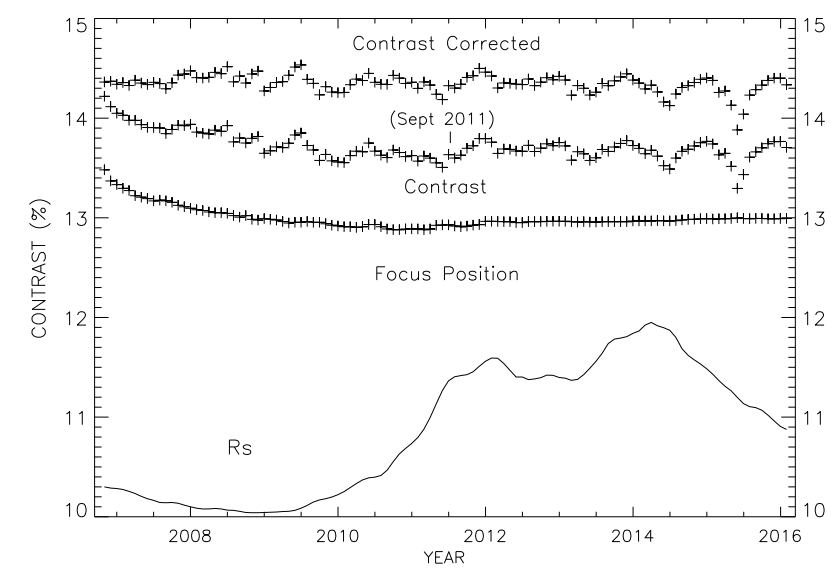

Fig. 4. Variations of the granulation contrast (middle), of the focus position transformed in contrast with the help of the linear relation in Fig. 3 (bottom), of the granulation contrast corrected by the variation of the focus position (top) and of the smoothed sunspot index. Each cross represents a monthly average. All variations are artificially shifted for clarity.

position has progressively and significantly drifted from its original position. However, when one considers short time interval, like a month during which our monthly analysis is made, the focus position is the same for all the selected images, while it departs from this nominal position for the discarded images. This confirms that the discarded images are blurred because of unaccurate focusing. The granulation contrast and the focus position of the SOT telescope are plotted in Fig. 3 (top) for the period November 2006-February 2016. As for the contrast, the plotted values for the focal length are monthly averages, but only for the selected images since the discarded images are not correctly focused. Both variations appear to have very similar long-term trends, including their stabilization after September 2011, confirming that the observed long-term variation of the granulation contrast is mainly of instrumental origin. In Fig. 3 (bottom) the contrast is plotted versus the focus position. The stars correspond to the period November 2006-December 2010, when both the contrast and the focus position decrease similarly: they clearly appear to be linearly related. The dispersion in the bulk of the plot is due to the yearly modulation of the contrast; the lower crosses correspond to the very deep last two minima of the annual modulation. The intensity of the blue continuum images has linearly decreased by a factor of 1.6 through the full period of observation, and therefore the transmission of the optical devices in this wavelength too. But the granulation contrast has not been much affected by this transmission reduction, since it didn't decrease anymore after the mirror temperature stabilization. The instrumental long-term variation can be corrected, at least partially during the period November 2006December 2010, thanks to the linear relation between the contrast and the focus position plotted in Fig. 3. First, the variation of the focus position is transformed in a variation of contrast of instrumental origin with the help of the linear relation. Then the observed contrast variation is divided by the instrumental variation normalized to one. Between January 2012 and February 2016 the focus position increased slightly, but because of the large yearly variation of the contrast, it is not possible to derive a linear relation between the two quantities, as is apparent in Fig. 3b, where they form the cloud of crosses. Consequently no focus correction is applied after January 2012. Moreover there is no contrast variation associated with the steep jump of the focus position between January 2011 and January 2012. No focus 


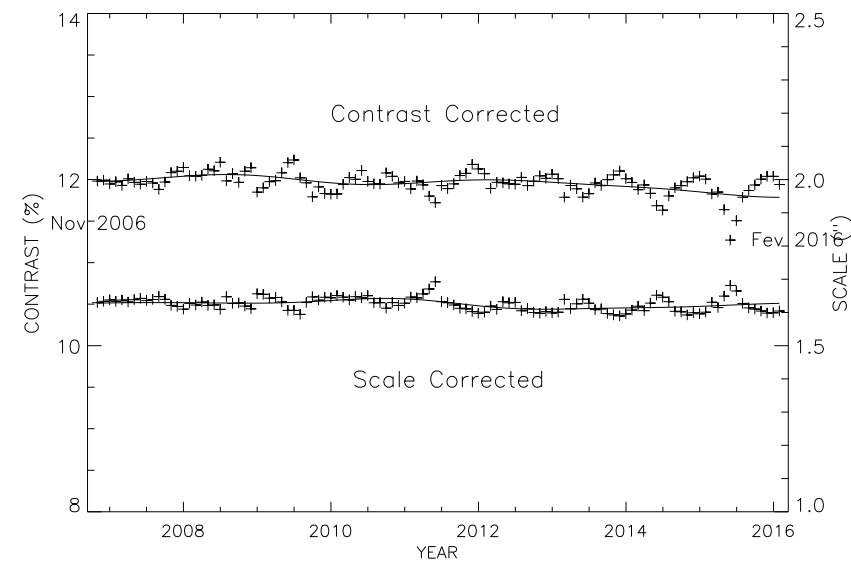

Fig. 5. Variations of the contrast and of the scale of the solar granulation corrected for the variation of the focus position, derived from images taken with the HINODE/SOT telescope between November 2006 and February 2016. Long-term variations obtained from FFT filtering are superimposed.

correction is made for this period neither. The corrected contrast variation is plotted in Fig. 4 together with the variation of the sunspot index: there appears to be no variation related to the solar cycle. In Fig. 5 the corrected contrast is plotted together with the variation of the scale corrected in the same way; longterm trends obtained from FFT filtering are superimposed to the corrected contrast and scale variations. Apparently there is no variation of the properties of the solar granulation related to the 11-yr cycle.

\section{Conclusion and discussion}

The HINODE/SOT blue-continuum images do not allow us to detect a variation of the properties of the granulation (contrast and scale) related to the solar cycle, either because the set of selected images is not homogeneous enough in term of spatial resolution and/or because the variation of the granulation is too weak. It is however interesting to know what amplitude of contrast and of scale variations of the solar granulation could have been detected with the HINODE/SOT images. For that purpose the observed variations of contrast and scale are modulated (multiplied) by a variation of increasing amplitude in phase with and proportional to the solar cycle. It is thus assumed that the amplitude of the variation of the granulation contrast is proportional to the smoothed sunspot index. The simulations for the contrast are shown in Fig. 6 with amplitudes between the minimum and the maximum of activity of $2 \% 3 \%, 5 \%$ and $10 \%$ of the nominal contrast $(12 \%)$. A solar cycle variation is clearly visible for $10 \%$ and $5 \%$, still a bit distinguishable for $3 \%$, but not at all for $2 \%$. Similar simulations are made with an 18 months positive and negative shift relative to the cycle, and also by dividing instead of multiplying in order to simulate antiphase variations, without or with shifts. The FFT filtered variations of modulated contrasts relative to their minimum value (which appear in the second half of 2009) are plotted in Fig. 7, for amplitudes of modulation $10 \%, 5 \%, 3 \%$ and $0 \%$, in order to show more clearly the effect of the modulation. The vertical dashed line separates the variation corrected by the focus variation on the left side, from the variation not corrected (because the mirror temperature was stabilized during this period) on the right side. The weak bump on the left side is probably an ill-corrected residual of non solar origin. On the right side, the amplitude of the maximum of contrast of the

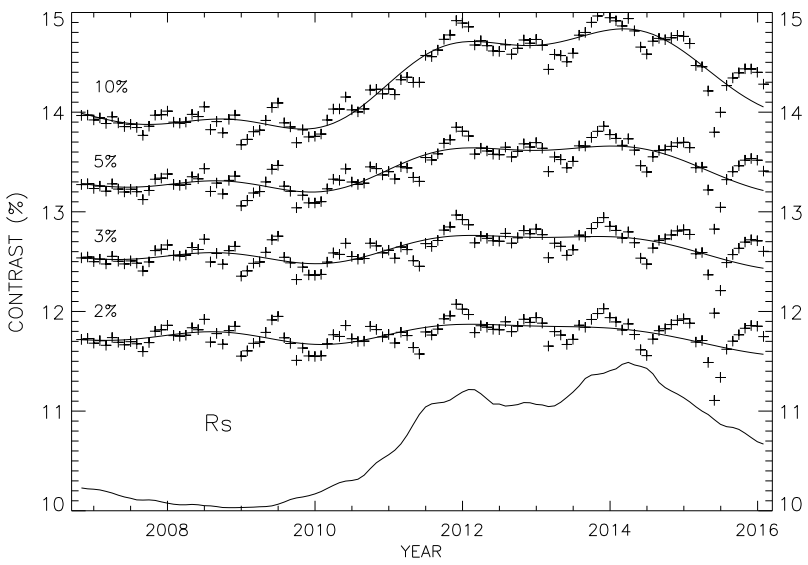

Fig. 6. Modulation of the observed variation of the granulation contrast modulated (multiplied) by a variation of increasing amplitude in phase with and proportional to the smoothed sunspot index Rs. Amplitudes between the minimum and the maximum of activity of $2 \%$ (bottom), $3 \%, 5 \%$ and $10 \%$ (top) of the nominal contrast (12\%) are shown, artificially shifted for clarity. Long-term variations obtained from FFT filtering are superimposed.

$3 \%$ modulation variation relatively to the minimum in 2009 is 2.8 times larger than that of the non solar bump, which can be regarded as noise. So, the signal to noise ratio is close to $3 \%$ and explains why a $3 \%$ modulation is detected by a simple visual inspection in Fig. 6. Thus in conclusion, the amplitude of the contrast variation of the solar granulation between the minimum of activity in 2009 and the maximum in 2014 was less than $3 \%$. We can also see in Fig. 7 that the observed contrast variation ( $0 \%$ modulation) exhibits a weak bump of amplitude $1.7 \%$ (of the nominal granulation contrast of 12\%) in the not focus corrected side, which is nearly in phase with the variation of the Sunspot Index. It thus could be of solar origin although its amplitude is well below the threshold of detectability of $3 \%$. Very similar results are obtained for the variation of the granulation scale. Concerning the meso- and supergranulation, Roudier et al. (2017) found no variation of their flow properties over the maximum of the solar cycle 24 between 2010 and 2015. Thus the turbulent convection does not appear to vary over all scales from granulation to supergranulation along the weak magnetic cycle 24 , or to vary too weakly to be detected with the available observations.

Muller et al. (2007) found that the contrast of the granulation varies in antiphase with the solar cycle between 1978 and 1993. The amplitude of the variation between the maxima of 1980 and 1991 and the minimum of 1986 was found as high as $20 \%$. But in this investigation, the contrast was derived from power spectra computed in logarithmic scale instead of in decimal scale like in the present paper. The comparison between the two ways of computing the contrast we made, shows that the "decimal contrast" is a little larger than the "logarithmic contrast". This means that the amplitude of the contrast variation in the Muller et al. (2007)'s paper, if computed with power spectra in decimal scale as in the present paper, would have been even larger than $20 \%$ between the maxima of the cycles 21 and 22 and the minimum between them in 1986. A question then arises: why was a variation detected during the period 1978-1993 with images taken at the Pic du Midi Observatory and not detected (or very weakly detected), in the period 2006-2016 with HINODE/SOT images from space? First it should be noted that the cycles 21 and 22 were two times stronger than the current cycle 24 . If the cause of a cyclic variation of the granulation contrast at the disk center is the level of the global magnetic activity, it would mean that 


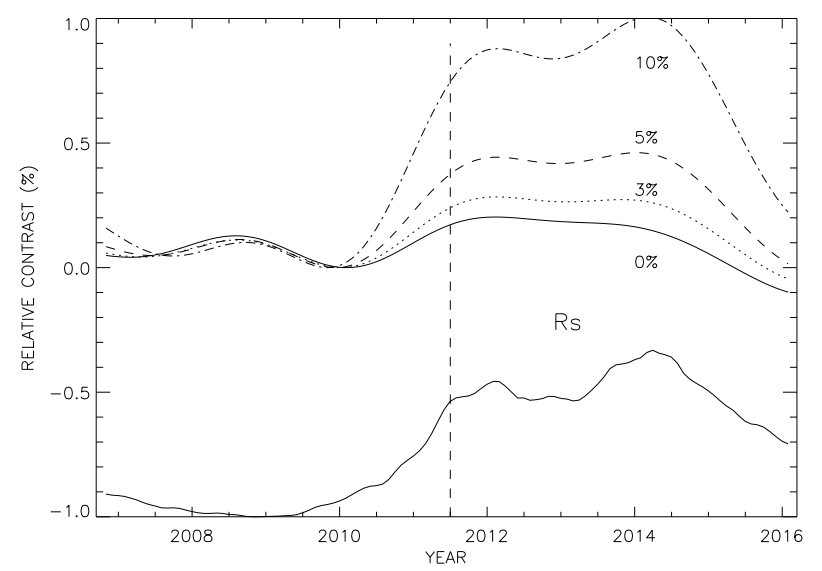

Fig. 7. FFT filtered modulated variations of the granulation contrast plotted relatively to the minima which appear in the second half of 2009. Compared to Fig. 6 the $2 \%$ amplitude modulation is replaced by a $0 \%$ modulation, which corresponds to the observed variation.

the effect of the global magnetic field on the convection is strong and non linear, since when the sunspot index increases for nearly zero to 110, like between 2009 and 2014, the contrast variation is less than $3 \%$, but it could be as high as $20 \%$ when the sunspot index increases from nearly zero to 220 like between 1986 and 1991. Another possibility is instrumental if the Pic du Midi set of images is not homogeneous enough, because of varying optical aberrations, atmospheric seeing, scattered light, and image digitization. But in that case, why is the variation not just at random instead of exhibiting a periodic variation in (anti)phase with the solar cycles 21 and 22? In contrast, no variation was detected for the scale, neither with the Pic du Midi nor with the HINODE/SOT images. One can also mention that, at the disk center, the variation of the number of magnetic bright points which form the photospheric network, varies by no more than $30 \%$ through the cycle 24 (Utz et al. 2016); this means that such a weak variation of the quiet sun network magnetic field has no detectable effect on the solar granulation observed with HINODE/SOT in the period November 2006-February 2016.

Thus, the question of the variation of the solar granulation related to the solar cycle is still open. To solve this issue, the granulation images should be restored from atmospheric seeing (for ground based observations) and corrected for instrumental aberrations like it was done, for example, by Bonet et al. (2004) on images taken with the Swedish 1-m telescope in La Palma. In the space, regular in-flight calibrations are recommended for further images restoration, as was done for the IMaX (Imaging Magnetograph eXperiment) instrument onboard the $1-\mathrm{m}$ stratospheric telescope of the SUNRISE mission (Vargas 2009). It would also be helpful if the next solar cycles are of higher amplitude than cycle 24. But this is out of human control.
Acknowledgements. The authors acknowledge the support of the AustrianFrench scientific exchange program "Amadeus" (R.M. and A.H.) and the support of the Austrian Fonds zur Förderung der Wissenschaftlichen Forschung (A.H. and D.U.). They are also grateful to the Hinode team for allowing them to use their data. Hinode is a Japanese mission developed and launched by ISAS/JAXA, collaborating with NAOJ as a domestic partner, NASA and STFC (UK) as international partners. Scientific operation of the Hinode mission is conducted by the Hinode science team organized at ISAS/JAXA. This team mainly consists of scientists from institutes in the partner countries. Support for the post-launch operation is provided by JAXA and NAOJ (Japan), STFC (U.K.), NASA (U.S.A.), ESA, and NSC (Norway). Part of this work was done while A.H. was visiting the Midi Pyrénées Observatory and R.M. the Institut für Geophysik, Astrophysik und Meteorologie, University Graz. The authors wish to thank the anonymous referee for very helpful comments and suggestions that improved the quality of the paper.

\section{References}

Basu, S., \& Antia, H. M. 2000, Sol. Phys., 192, 449

Bonet, J. A., Márquez, I., Muller, R. Sobotka, M., \& Tritschler, A. 2004, A\&A, 423, 737

Buehler, D., Lagg, A., \& Solanki, S. 2013, A\&A 555, A33

Burtseva, O., Hill, F., Kholikov, S., \& Chou,, D. Y. 2009, Sol. Phys., 258, 1

Cattaneo, F., Emonet, T., \& Weiss, N. 2003, ApJ, 588, 1183

De Rosa, M. L., Gilman, P. A., \& Toomre, J. 2002, ApJ, 581, 1356

Dziembowski, W. A., \& Goode, P. R. 2005, ApJ, 625, 548

Emilio, M., Bush, R. I., Kuhn, J. R., \& Scherrer, P. 2007, ApJ, 660, L161

Fröhlich, C. 2000, Space Sci. Rev., 94, 15

Hathaway, D. H., Beck, J. G., Bogart,R. S., Bachmann, K. T., et al. 2000, Sol. Phys., 193, 299

Houdek, G., Chaplin, W. J., Appourchaux, J., et al. 2001, MNRAS, 327, 483

Kuhn, J. R., \& Schüssler, M. 2000, Space Sci. Rev., 94, 117

Kuhn, J. R., Libbrecht, K. G., \& Dicke, R. H. 1988, Science, 242, 908

Lefebvre, S., \& Kosovichev, A. G. 2005, ApJ, 633, 149

Leighton, R., Noyes, R., \& Simon, G. 1962, ApJ, 135, 474

Li, L. H., Basu, S., Sofia, S., Robinson, F. J., \& Demarque, P. 2003, ApJ, 591, 1267

Li, L. H., Sofia, S., Ventura, P., et al. 2009, ApJS, 182, 584

Libbrecht, K. G., \& Woodard, M. F. 2000, Nature, 345, 779

Macris, C., \& Rösch, J. 1983, C. R. Acad. Sci. Paris, 296, 265

Macris, C., Muller, R., Rösch, J., Roudier, T. 1984, in Small Scale Dynamical Processes in Quiet Stellar Atmospheres, ed. S. K. Keil, 265

Mullan, D. J., MacDonald, J., \& Townsend, R. H. D. 2007, ApJ, 670, 1420

Muller, R. 1988, Adv. Space Res., 8, 159

Muller, R., Hanslmeier, A., \& Saldana-Munoz, M. 2007, A\&A, 475, 717

Muller, R., Utz, D., \& Hanslmeier, A. 2011, Sol. Phys., 274, 87

Nordlund, A., Stein, R. F., \& Asplund, M. 2009, Liv. Rev. Sol. Phys., 6, 2

November, L. J., \& Simon, G. 1988, ApJ, 333, 427

Parker, E. N. 1995, ApJ, 440, 415

Penza, V., \& Del Moro, D. 2009, Mem. Soc. Astron. It., 80, 282

Rieutord, M., Meunier, N., Roudier, T., Rondi, S., et al. 2008, A\&A, 479, L17

Roudier, T. 1986, PhD Thesis, University Paul Sabatier, Toulouse, France

Roudier, T., \& Muller, R. 1986, Sol. Phys., 107, 11

Roudier, T., Malherbe, J. M., \& Mirouh, G. M. 2017, A\&A, 598, A99

Solanki, S. K., Krivova, N. A., \& Haigh, J. D. 2013, ARA\&A, 51,311

Tsuneta, S., Suematsu, Y., Ichimoto, K., Shimizu, T., et al. 2008, Sol. Phys. 249,167

Utz, D., Muller, R., Thonhofer, S., Veronig, A. et al. 2016, A\&A, 585, A39

Vargas Dominguez, S. 2009, PhD Thesis, University of La Laguna, Spain

Vögler, A., Sheylag, S., Schüssler, M., Cattaneo, F., et al. 2005, A\&A, 429, 335

Wilson, R. C., \& Hudson, H. S. 1991, Nature, 351, 42 